\title{
The use of Tranexamic Acid in Total Elbow Replacement to Reduce Post-Operative Wound Infection
}

\author{
Syed Mannan, Mohammed Ali ${ }^{凶}$, Lukasz Mazur, Mei Chin, Ahmed Fadulelmola \\ North Cumbria University Hospital NHS Trust \\ $\triangle$ Corresponding author: mohammed.ali17@nhs.net \\ (C) Ivyspring International Publisher. This is an open access article distributed under the terms of the Creative Commons Attribution (CC BY-NC) license \\ (https://creativecommons.org/licenses/by-nc/4.0/). See http://ivyspring.com/terms for full terms and conditions.
}

Received: 2018.02.19; Accepted: 2018.04.23; Published: 2018.05.28

\begin{abstract}
Background: Incidence of infection following total elbow replacement (TER) is recognised to be higher compared to hip or knee arthroplasty. Extensive swelling following TER can complicate the wound healing which might lead to infection. Tranexamic Acid (TXA) is proven to reduce blood loss peri-operatively which might contribute to better healing outcomes. Our aim is to assess the effect of TXA in wound healing following TER.

Methods: A retrospective review of a single surgeon case series. 10 patients had TER mainly for complicated elbow fractures, four of them were relatively immune-supressed. All patients had 2 grams of TXA and antibiotics intra-operatively. All were reviewed at two weeks following surgery for wound check and removal of surgical clips.

Results: Seven females and three males with a mean age of 81.5 had TER and TXA. The mean level of pre-operative haemoglobin was $134.40 \mathrm{~g} / \mathrm{l}$ and the mean post-operative level was $122.70 \mathrm{~g} / \mathrm{l}$. No patient in this series required blood transfusion. At two weeks and six weeks follow-up, all wound healed up with no signs of infection.

Conclusion: TXA has been proven to be safe an effective way of reducing peri-operative bleeding. TXA maintains haemostasis after releasing the tourniquet and therefore reduces the swelling and wound complications post-operatively
\end{abstract}

\section{Introduction}

Total elbow replacement (TER) has become a good alternative surgical solution for a large percentage of patients with significant joint damage. TER has proven to be able to overcome the previous difficulties faced by fixation as it provides good stability and allows early mobilisation. Furthermore, TER has a speedier rehabilitation course and much better functional outcomes especially in elderly low-demand patients with osteopenia or osteoporosis (1). However, the incidence of infection, nerve damage, implant loosening and periprosthetic fracture cannot be considered negligible, as it seems to be higher compared to hip or knee arthroplasty. Impaired wound healing possibly can lead to deep infection (2) which is a devastating complication, with an incidence ranging from 1 to 9 percent (3). Reasons for wound healing problems may be insufficient blood flow in the wound margins caused by an incorrect skin incision or extensive soft tissue dissection. Furthermore, extensive swelling can lead to skin necrosis which can predispose to infection (4). Anti-fibrinolytic agents such as Tranexamic Acid (TXA) can minimise the blood loss, post-operative soakage, seroma and swelling in patients with both normal and exaggerated fibrinolytic responses to surgery and trauma avoiding increasing the risk of postoperative or post-injury complications $(5,6,7)$. As a synthetic derivative of lysine with a molecular weight of $157 \mathrm{~g} / \mathrm{mol}$, TXA employs its anti-fibrinolytic effect by a reversible interaction with 
plasminogen and the active protease, plasmin (8). TXA can be administered through both intravenous or topical /infiltration routes and majority of published research revealed both ways to be effective (8). At the cellular level, fibrin is constantly deposited and eliminated by fibrinolytic mechanisms. Tranexamic acid inhibits proteolytic degradation of fibrin by blocking the attachment of plasminogen and plasmin (9). Kwaan and Astrup (10) have previously showed, in skin wounds, that antifibrinolytic agents increase collagen synthesis and tensile strength within granulation tissue, presumably by preserving the fibrin matrix. Add to that, Vinckier's study concluded that tranexamic acid, by stabilizing the fibrin structures within the non- collapsible dental socket, can even accelerate normal wound healing (11).

In this case series, we retrospectively review the early outcomes following use of TXA in total elbow arthroplasty. We report the blood loss, wound complications and range of movement intra and 2 weeks post-operatively. This case series has been reported in line with the PROCESS criteria (12).

\section{Methods}

Retrospectively, we reviewed ten consecutive patients who had tranexamic acid for total elbow arthroplasty between January 2016 to June 2017 in a district general hospital. The study comprised review of patients' clinical notes and radiographs. When there was no contra-indication, patient was given two grams of Tranexamic Acid intra-operatively by the senior author. Operations were all performed by a senior upper limb consultant under general anaesthesia. Operations were performed in a supine position with the arm placed on the patient's chest. A pneumatic tourniquet was used in all cases. Using a posterior midline incision and Triceps-on approach to the elbow joint, the joint was exposed, prepared and replaced.

For antimicrobial prophylaxis, intravenous Tiecoplanin and Gentamycin were given on anaesthetic induction as per hospital policy. The operated arm of all patients had a wool and crepe dressing and put in a broad arm sling post-operatively.

All patients had post-operative check radiographs of the elbow. Physiotherapy referral initiated immediately post-operatively as per our local hospital protocol. Patients were allowed immediate post-operative gentle full range of movement, non-weight bearing as the pain allows. Patients were followed-up two weeks after surgery in outpatient clinics, where they had a wound check, removal of skin clips and range of movement evaluation.
In accordance with the declaration of Helsinki, the research registry number is: 3585 . Ethical approval was not needed for this case series as this is a retrospective analysis with no intervention resulting in the deviance of standard clinical practice. Fully anonymised data.

\section{Results}

The mean age was 81.5 years (range 74-94). Seven patients were females $(70 \%)$ and three were males (30\%) (Table 1$)$.

Table 1. Patients demographics

\begin{tabular}{llll}
\hline Age & Gender & $\begin{array}{l}\text { AO } \\
\text { Classification }\end{array}$ & \multicolumn{2}{l}{ Past medical history } \\
\hline 87 & F & B2 & Chronic kidney disease, Hypertension \\
87 & M & A2 non-union & Osteoarthritis, Benign prostatic hyperplasia \\
81 & F & Arthritis & Rheumatoid arthritis, Osteoporosis \\
79 & F & A2 non-union & Diabetes type-2, Chronic kidney disease \\
81 & M & A2 non-union & Diabetes type-2, Chronic kidney disease, \\
& & & Osteoarthritis \\
88 & F & A2 non-union & Alzheimer's, Hypertension \\
74 & F & C3 & COPD, Osteoporosis \\
90 & F & C3 & Alzheimer's, Osteoporosis, arthritis \\
74 & M & C3 & No significant PMH \\
78 & F & C3 & Osteoarthritis \\
\hline
\end{tabular}

According to the $\mathrm{AO}$ classification, four patients $(40 \%)$ sustained type A2 fracture. four $(40 \%)$ had type C3 and one (10\%) was B2. There was only one non-trauma patient $(10 \%)$ with severe RA (Figures 1 and 2).

All the trauma patients had a CT scan of the elbow with 3D reconstruction to allow better evaluation of the injury (Figure 3).

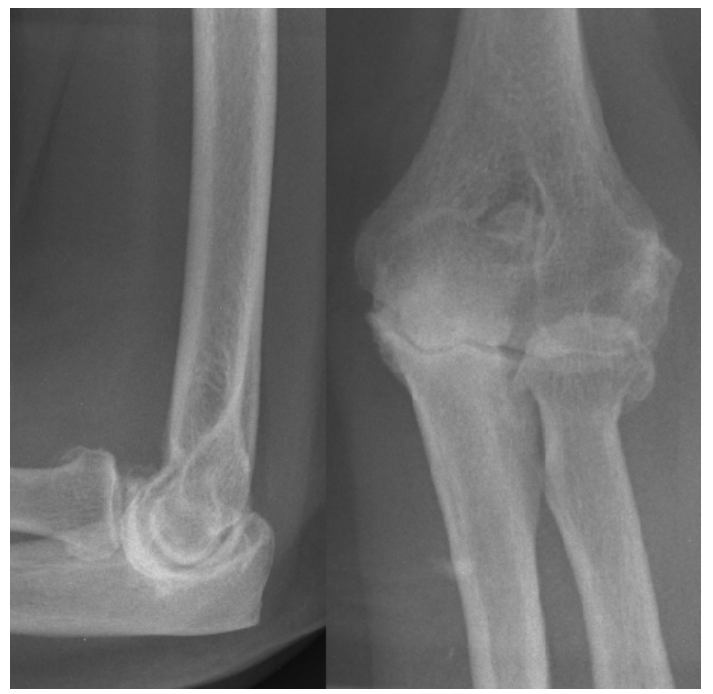

Figure 1: Plain elbow radiographs show sever arthritic changes 


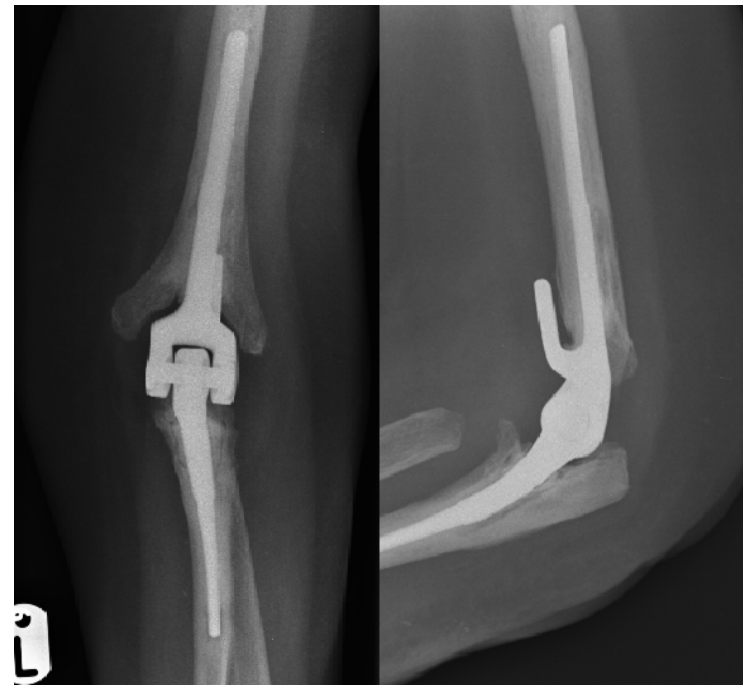

Figure 2: Total elbow replacement.

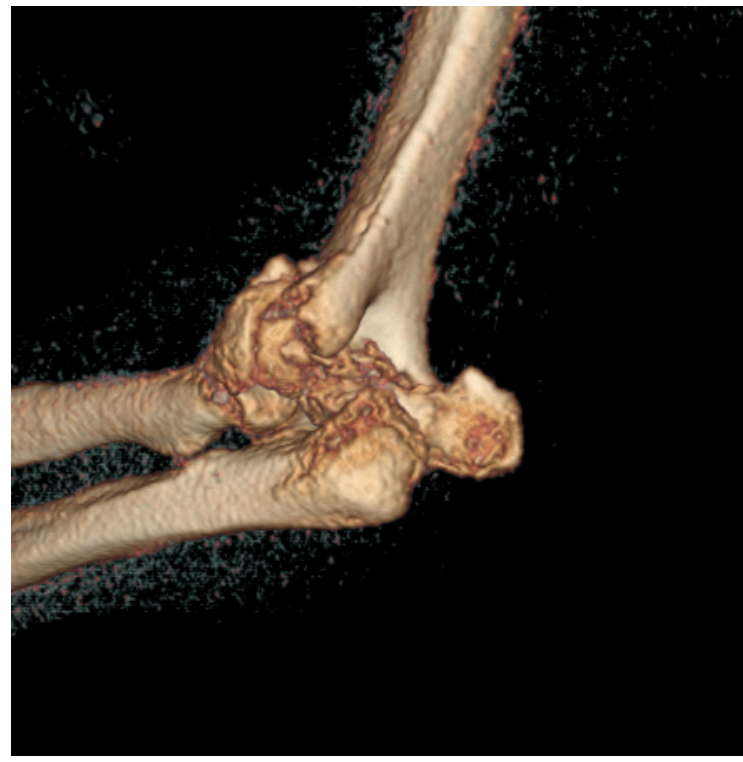

Figure 3: 3D reconstruction shows $A O$ type $C 3$ fracture.

Operative notes of those patients described five fractures $50 \%$ to be comminuted fracture, three $(30 \%)$ were trans-condylar and one was supra-condylar fracture. The mean tourniquet time was 94.85 minutes (range 69-120). All 10 (100\%) of patients had 2grams of TXA and all were given antibiotics intra-operatively according to our hospital policy. The mean level of pre-operative haemoglobin was $134.40 \mathrm{~g} / 1$ (range 117-166) and the mean post-operative level was $122.70 \mathrm{~g} / 1$ (range 99-144). No patient in this series required blood transfusion. six patients $(60 \%)$ had achieved full range of motion intra-operatively and four $(40 \%)$ had full flexion and 10-20 degrees of extension lag. At two weeks post-operatively, all patient's records reported the same range of movement achieved intra-operatively. Although, four patients in this series were considered to have high chances of wound complication (two diabetics, one with COPD and one with RA), at two weeks, all patients' wounds healed up and had their surgical clips removed. No patient developed wound dehiscence or wound infection. Patients were reviewed again at six weeks when the wounds were again examined and there were no complications reported. In this series, we had no incidents of deep venous thrombosis among our patients nor any other complications related to TXA.

\section{Discussion}

Post-operative infection remains one of the major concerns in the field of arthroplasty. The percentage of periprosthetic infection in elbow arthroplasty is reported to be higher than in other joints. Currently, the incidence of peri-prosthetic infection is estimated to be between $1 \%$ and $9 \%(3,13)$. Voloshin et al $(14)$ and Gschwend et al (15), conducted two separate literature reviews studying complication following total elbow arthroplasty. the two reviews concluded that the most frequent complications include loosening, instability, and infection. This is believed to be multifactorial, considering fragility of soft tissues, lower immunity accompanying rheumatoid arthritis and the chances of contamination in trauma cases. Ewald et al in 1993 (16) compared the outcomes of lateral approach with posterior approach. They concluded that lateral approach carries lower risk of wound complications. Ljung et al (17) used the lateral approach in their series and they experienced wound healing complications in $4 \%$. Furthermore, some authors advocated postoperative immobilization as it reduces wound healing complications following total elbow arthroplasty $(18,19)$. In the study by Ljung $(17)$, no wound healing complications were reported the group with longer period of immobilisation (12 days).

Although the use of tourniquet in TER will significantly reduce intraoperative blood loss, internal bleeding after releasing the tourniquet comes with an increasing risk of post-operative swelling, pain and wound complications. Currently, most of hip and knee surgeons are routinely using TXA to minimise the amount of intra-operative bleeding. Guerreiro et al (20) conducted a randomised trial investigating the role of TXA in total knee arthroplasty. Topical TXA was applied to 22 patients before closure of the joint capsule. In addition to reducing bleeding, TXA improved pain and increased flexion gain in the first hours after TKA. Although some studies suggested that TXA may have adverse effects on tendon healing (21), several authors advocated the positive role of TXA on wound healing and skin regeneration. Björlin et al (22) studied the effect of epsilon-aminocaproic 
acid and TXA on wound healing in white rats. Wounds injected with tranexamic acid had a higher tensile strength than those pre-treated with epsilonaminocaproic acid. Even compared at the same ionic strength, similar results were obtained. Björlin concluded that TXA has a positive effect on wound healing, and that this effect is not due to the anti-fibrinolytic properties, as both are powerful fibrinolytic inhibitors. Furthermore, Gupta et al (23) in 2015 studied the role of TXA in prevention of wound infection in postoperative orthopaedic patients. The study included 120 operations including; spine, intertrochanteric fractures, hip hemiarthroplasty and general trauma. Two patients out of 60 on TXA were infected whereas 4 out of 60 patients who were not given TXA were infected. They also noted that TXA is effective in reducing wound dehiscence rate, need of prolonged antibiotics. In 2014, Yuan (24) conducted a study to determine whether TXA could repair the skin barrier by means of tight intercellular junctions using damaged skin models. using bioengineering technology and immunohistochemistry tests, they concluded that TXA can accelerate skin barrier recovery and upregulate occludin induced by physicochemical damages of human skin.

In upper limb arthroplasty, TXA is commonly administered when performing a shoulder procedure. However, because of using tourniquet it is not gaining popularity in the elbow arthroplasty world hence there is no evidence in the literature. We think it is very appropriate to use a haemostatic agent to guard against these expected complications. Intravenous tranexamic acid is found to significantly reduce peri-operative blood loss and blood transfusion requirements after arthroplasty, without an apparent increase in harm. We acknowledge that our retrospective study has limitations including the small sample size, lack of another arm for comparison, and considering other variables such as pain and functional outcome scores.

\section{Conclusion}

TXA has been proven to be safe and effective way of reducing peri-operative bleeding. TXA maintains haemostasis after releasing the tourniquet and therefore reduces the swelling and wound complications post-operatively. Studies with bigger sample size are needed in the future to investigate the role of TXA in wound healing following TER.

\section{Competing Interests}

The authors have declared that no competing interest exists.

\section{References}

1. McKee MD, Veillette CJ, Hall JA, et al. A multicenter, prospective, randomized, controlled trial of open reduction-internal fixation versus total elbow arthro- plasty for displaced intra-articular distal humeral fractures in elderly patients. J Shoulder Elbow Surg 2008.

2. Ljung $\mathrm{P}$, Bornmyr $\mathrm{S}$, Svensson $\mathrm{H}$. Wound healing after total elbow replacement in rheumatoid arthritis. Wound complications in 50 cases and laser-Doppler imaging of skin microcirculation. Acta Orthop Scand. 1995 Feb;66(1):59-63.

3. Ewald F C, Simmons E D, Sullivan J A, Thomas W H, Scott R D, Poss R, Thornhill T S, Sledge C B. Capitello- condylar total elbow replacement in rheumatoid arthri- tis. Long-term results. J Bone Joint Surg (Am) 1993; 75: 498-507.

4. Li Y, Bao R-H, Jiang Z-Q, Wu H-Y. Complications in operative fixation of calcaneal fractures. Pakistan Journal of Medical Sciences. 2016;32(4):857-862

5. Ishida K, Tsumura N, Kitagawa A, Hamamura S, Fukuda K, Dogaki Y, Kubo S,Matsumoto T, Matsushita T, Chin T, Iguchi T, Kurosaka M, Kuroda R. Intra-articular injection of tranexamic acid reduces not only blood loss but also knee joint swelling after total knee arthroplasty. Int Orthop. 2011 Nov;35(11):1639-45.

6. Huang F, Wu D , Ma G, Yin Z, Wang Q. The use of tranexamic acid to reduce blood 1 oss and transfusion in major orthopedic surgery: a meta - analysis. J Surg Res. 2014 Jan; 186 (1): 318 - 27

7. Krohn CD,Sørensen R, Lange JE, Riise R, Bjørnsen S, Bross tad F. Tranexamic acid given into the wound reduces postoperative blood loss by half in major orthopaedic surgery. Eur J Surg Suppl. 2003 Jul; (588): 57 - 61.

8. Kim C, Park SS-H, Davey JR. Tranexamic acid for the prevention and management of orthopedic surgical hemorrhage: current evidence. Journal of Blood Medicine. 2015;6:239-244.

9. Wiman B and Collen D. Molecular Mechanism of Physiological Fibrinolysis. Nature 1978;272:549-550.

10. Kwaan H.C and Astrup T. Tissue Repair in Presence of Locally Applied Inhibitors of Fibrinolysis. Exp Mol Pathol 1969;11:82-88

11. Vinckier F, Vermylen J. Wound healing following dental extractions in rabbits:effects of tranexamic acid, warfarin anti-coagulation, and socket packing. J DentRes. 1984 May;63(5):646-9.

12. Riaz Agha A, et al. Preferred reporting of case series in surgery; the PROCESS guidelines. Int J Surg. 2016.

13. Yamaguchi K, Adams RA, Morrey BF. Infection after total elbow arthroplasty. J Bone Joint Surg Am. 1998;80:481-91.

14. Voloshin I, Schippert DW, Kakar S, Kaye EK, Morrey BF. Complications of total elbow replacement: a systematic review. J Shoulder Elbow Surg. 2011 Jan;20(1):158-68.

15. Gschwend N, Simmen BR, Matejovsky Z. Late complications in elbow arthroplasty. J Shoulder Elbow Surg 1996;5:86-96.

16. Ewald F C, Simmons E D, Sullivan J A, Thomas W H, Scott R D, Poss R, Thornhill T S, Sledge C B. Capitello- condylar total elbow replacement in rheumatoid arthri- tis. Long-term results. J Bone Joint Surg (Am) 1993; 75: 498-507.

17. Ljung $\mathrm{P}$, Bornmyr S, Svensson $\mathrm{H}$. Wound healing after total elbow replacement in rheumatoid arthritis. Wound complications in 50 cases and laser-Doppler imaging of skin microcirculation. Acta Orthop Scand. 1995 Feb;66(1):59-63.

18. Brady 0, Quinlan W. The Guildford elbow. J Hand Surg (Br) 1993; 18: 389-93.

19. Maloney W J, Schurman D J. Cast immobilization after total elbow arthroplasty.A safe cost-effectivemethod of initial postoperative care. Clin Orthop 1989; 245: 117-22.

20. Guerreiro JPF, Badaro BS, Balbino JRM, Danieli MV, Queiroz AO, Cataneo DC. Application of Tranexamic Acid in Total Knee Arthroplasty Prospective Randomized Trial. Open Orthop J. 2017 Aug 29;11:1049-1057.

21. Çıraklı A, Gürgör PN, Uzun E, Erdem H, Çankaya S, Baş O. Local application of tranexamic acid affects tendon healing negatively in the late period. Eklem Hastalik Cerrahisi. 2018 Apr;29(1):20-6.

22. Björlin G, Nilsson IM. The effect of antifibrinolytic agents on wound healing. Int J Oral Maxillofac Surg. 1988 Aug;17(4):275-6.

23. Gupta A, et al. Post-Surgical Wound Care in Orthopedics: Role of Tranexamic Acid. Journal of Evolution of Medical and Dental Sciences 2015; 4: 5716-5720.

24. Yuan C, Wang XM, Yang LJ, Wu PL. Tranexamic acid accelerates skin barrier recovery and upregulates occludin in damaged skin. Int J Dermatol. 2014 Aug;53(8):959-65. 